\title{
Acute Myocardial Infarction in an Adolescent Receiving Anagrelide for Essential Thrombocythemia with Underlying Persistent Coronary Endothelial Dysfunction
}

Ryuhei Tanaka, ${ }^{1} \mathrm{MD}$, Yodo Gatate ${ }^{2} \mathrm{MD}$, Yasumori Sujino, ${ }^{2} \mathrm{MD}$, Maki Todo, ${ }^{3} \mathrm{MD}$, Atsuhiko Ohta, ${ }^{1} \mathrm{MD}$ Yuki Shimizu, ${ }^{1}$ MD, Atsuko Watanabe, ${ }^{1} \mathrm{MD}$, Ken Naganuma, ${ }^{1} \mathrm{MD}$, Toshihiro Muramatsu, ${ }^{2} \mathrm{MD}$, Kenji Fukushima, ${ }^{4} \mathrm{MD}$ and Shintaro Nakano, ${ }^{2} \mathrm{MD}$

\begin{abstract}
Summary
Essential thrombocythemia (ET) is a Philadelphia chromosome-negative myeloproliferative disorder that is characterized by the overproduction of platelets and a marked increase in the numbers of mature megakaryocytes present in the bone marrow. Thrombohemorrhagic disorders are major morbidities of ET, especially those with mutations in the gene encoding Janus kinase 2 (JAK2). In this study, we report the case of an 18-year-old patient with ET carrying JAK2 mutation who developed acute ST-elevation myocardial infarction (STEMI) 5 months after a commencement of anagrelide. Coronary endothelial dysfunction confirmed by positive acetylcholine provocation test lasted a year after the occurrence of STEMI. Furthermore, intracoronary imaging using optical coherence tomography demonstrated non-atheromatous intimal fibrosis possibly due to chronic endothelial damage. The coronary pathologies reflected chronic change potentially associated with properties of ET and JAK2 mutation in addition to hyperviscosity. These observations suggest that the side effect of anagrelide in our patient was considered causative, while underlying chronic endothelial dysfunction and adverse endothelial remodeling may be predisposing factors to his fatal cardiovascular events.
\end{abstract}

Key words: Oncocardiology, Acute coronary syndrome

(Int Heart J 2020; 61: 1289-1293)

E ssential thrombocythemia (ET) is a form of myeloproliferative neoplasms. It is a clonal hematopoietic disorder that is characterized by markincreased clusters of hyperlobulated mature megakaryocytes in the bone marrow and by sustained thrombocytosis of peripheral blood. ${ }^{1,2)}$ Patients with ET, especially those with mutations in the gene encoding Janus kinase 2 (JAK2), may experience cardiovascular complications, such as arterial/venous thrombosis or hemorrhages, due to multifactorial etiologies. ${ }^{3)}$ Anagrelide is a non-leukemogenic cytoreductive drug used for the treatment of ET in patients with a long life expectancy; however, this drug has been associated with various cardiovascular side effects, such as palpitation, atrial fibrillation, congestive heart failure, coronary artery disease, and arterial hypertension. ${ }^{4-6)}$

In this study, we report the case of an adolescent patient with ET carrying JAK2 mutation who suffered from ST-segment elevation myocardial infarction (STEMI) occurring after the initiation of anagrelide treatment. Persistent nitroglycerin-responsive coronary vasospasm and non- atheromatous intimal fibrosis potentially related to properties of ET and JAK2 were considered as underlying etiologies for his susceptibility to STEMI.

\section{Case Report}

An 11-year-old Japanese boy was referred to the pediatric hematology/oncology department of our institution for investigation of increased platelet count $\left(1273 \times 10^{9} /\right.$ L). The diagnosis of ET was made based on the hematological criteria, such as abnormal bone marrow findings (Supplemental Figure 1), identification of the JAK2 V617F mutation, and absence of the BCR-ABL1 fusion gene. ${ }^{1)} \mathrm{He}$ had suffered from intractable headache; this presumable vasomotor symptom was successfully ameliorated by administration of low-dose aspirin $(100 \mathrm{mg}$ daily). However, his platelet count remained high for years, reaching $1250 \times 10^{9} / \mathrm{L}$ by the age of 18 when he presented an intramuscular hemorrhage in the right buttock. As he was assumed to be at high risk for thrombohemorrhagic events, ${ }^{5,78}$ non-leukemogenic cytoreductive

From the ${ }^{1}$ Department of Pediatric Hematology/Oncology, Saitama Medical University International Medical Center, Saitama, Japan, ${ }^{2}$ Department of Cardiology, Saitama Medical University International Medical Center, Saitama, Japan, ${ }^{3}$ Department of Pharmacy, Saitama Medical University International Medical Center, Saitama, Japan and ${ }^{4}$ Department of Nuclear Medicine, Saitama Medical University International Medical Center, Saitama, Japan.

Address for correspondence: Ryuhei Tanaka, MD, Department of Pediatric Hematology/Oncology, Saitama Medical University International Medical Center, 1397-1 Yamane, Hidaka, Saitama 350-1298, Japan. E-mail: rytanaka@saimata-med.ac.jp or Shintaro Nakano, MD, Department of Cardiology, Saitama Medical University International Medical Center, Saitama, Japan. E-mail: snakano@saitama-med.ac.jp

Received for publication May 28, 2020. Revised and accepted August 11, 2020.

Released in advance online on J-STAGE November 13, 2020.

doi: 10.1536/ihj.20-377

All rights reserved by the International Heart Journal Association. 

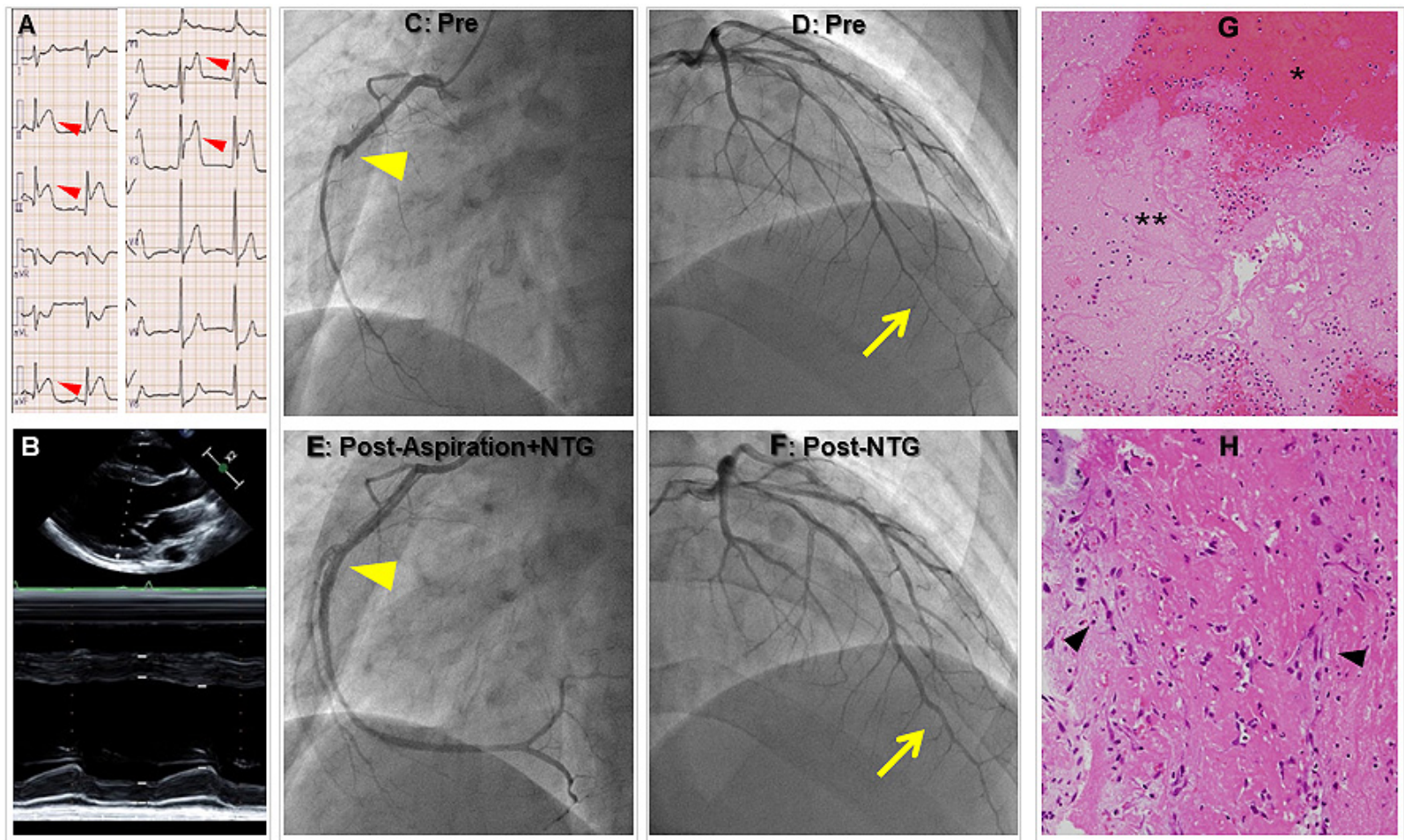

Figure 1. Findings at the onset of acute myocardial infarction. A: Electrocardiogram showing sinus rhythm and prominent ST-T elevation in leads II, III, aVF, and V1-V3 (arrowheads) with reciprocal change. B: Echocardiography showing left ventricular (LV) ejection fraction of 46.7\%, LV end-diastolic (LVEDD) and end-systolic dimension (LVESD) of 59/45 mm, with akinetic inferoseptal-inferior area from the base to apex and severely hypokinetic anterioseptal area at the base and middle level. C and D: Coronary angiograms before intervention showing occlusion of the right coronary artery (RCA, arrowhead) and spastic left anterior descending artery (LAD, arrow). E and F: Recanalized RCA by thrombus aspiration and intracoronary nitroglycerin infusion and dilated LAD with nitroglycerin infusion only (arrow). G and H: Hematoxylin-eosin staining demonstrated thrombus with clusters of red blood cells $(*)$ surrounded by fibrin accumulations $(* *)$, some inflammatory cells, and fibroblastic cells (arrowheads).

therapy with anagrelide (1.0 mg daily $)^{9)}$ was commenced, after confirming normal cardiac function at baseline (Supplemental Figure 2). Anagrelide successfully reduced the platelet (range, $500-700 \times 10^{9} / \mathrm{L}$ ) and neutrophil count ranged within the normal limit, and aspirin was stopped 2 months after the initiation of anagrelide.

However, the patient began to feel periodic palpitations or vague chest sensations a month after the initiation of anagrelide, although no abnormal 12-lead/24-hour electrocardiographic changes were documented. Five months after the commencement of anagrelide, the patient was transported to our cardiac center due to intermittent but progressively worsening precordial discomfort. Upon arrival, he was conscious with a body temperature of $36.5^{\circ} \mathrm{C}$, heart rate of 87 beats/minute, and systolic/diastolic pressure of $133 / 87 \mathrm{mmHg}$. Auscultation revealed no rales or abnormal heart sounds except for marginal third sounds. Cardiomegaly and mild pulmonary edema were identified on chest radiography. The electrocardiogram showed sinus rhythm and prominent ST-T elevation in leads II, III, aVF, and V1-V3 with reciprocal change (Figure 1A). Echocardiography showed left ventricular dysfunction with abnormal segmental wall motion (Figure 1B). Blood tests revealed leukocytosis (white blood cell count of $14.7 \times 10^{9} /$ L with neutrophils $81.5 \%$, hemoglobin $15.1 \mathrm{~g} / \mathrm{dL}$, and he- matocrit $45.0 \%$ ), thrombocytosis (platelet count $894 \times 10^{9} /$ $\mathrm{L}$ ), elevated markers for cardiac injury (serum troponin I $1288 \mathrm{pg} / \mathrm{mL}$ [upper limit of normal, $26.2 \mathrm{pg} / \mathrm{mL}$ ], creatine-kinase $274 \mathrm{IU} / \mathrm{L}$ [244 IU/L], and creatine-kinase MB isozyme $16.4 \mathrm{ng} / \mathrm{mL}[<5 \mathrm{ng} / \mathrm{mL}]$ ), almost normal hepatic and renal markers, and negative for thrombophilia markers (see details in Supplemental Table). He had no history of smoking. Emergent coronary angiography revealed total occlusion of the middle right coronary artery (RCA) and spastic stenosis of the distal left anterior descending artery (LAD) (Figure 1C and D). The RCA was successfully recanalized by thrombus aspiration and intracoronary nitroglycerin infusion, whereas the spastic LAD was dilated with the intracoronary nitroglycerin infusion only (Figure $1 \mathrm{~F}$ and $\mathrm{G}$, maximum serum creatine-kinase $9734 \mathrm{IU} / \mathrm{L}$ and creatine-kinase MB isozyme $600 \mathrm{ng} / \mathrm{mL}$, histological findings in Figure $1 \mathrm{H}$ and I). ${ }^{99 \mathrm{~m}}$ Technetiumpyrophosphate scintigraphy was performed 3 days later and cardiac magnetic resonance imaging 10 days later, which demonstrated extensive, partially irreversible myocardial infarction in the broad inferior and apical anterior wall (Figure 2).

Anagrelide, a potential causative agent for the observed coronary event, was ceased. Instead, hydroxyurea (1000 mg daily) and low-dose aspirin (100 mg daily) 

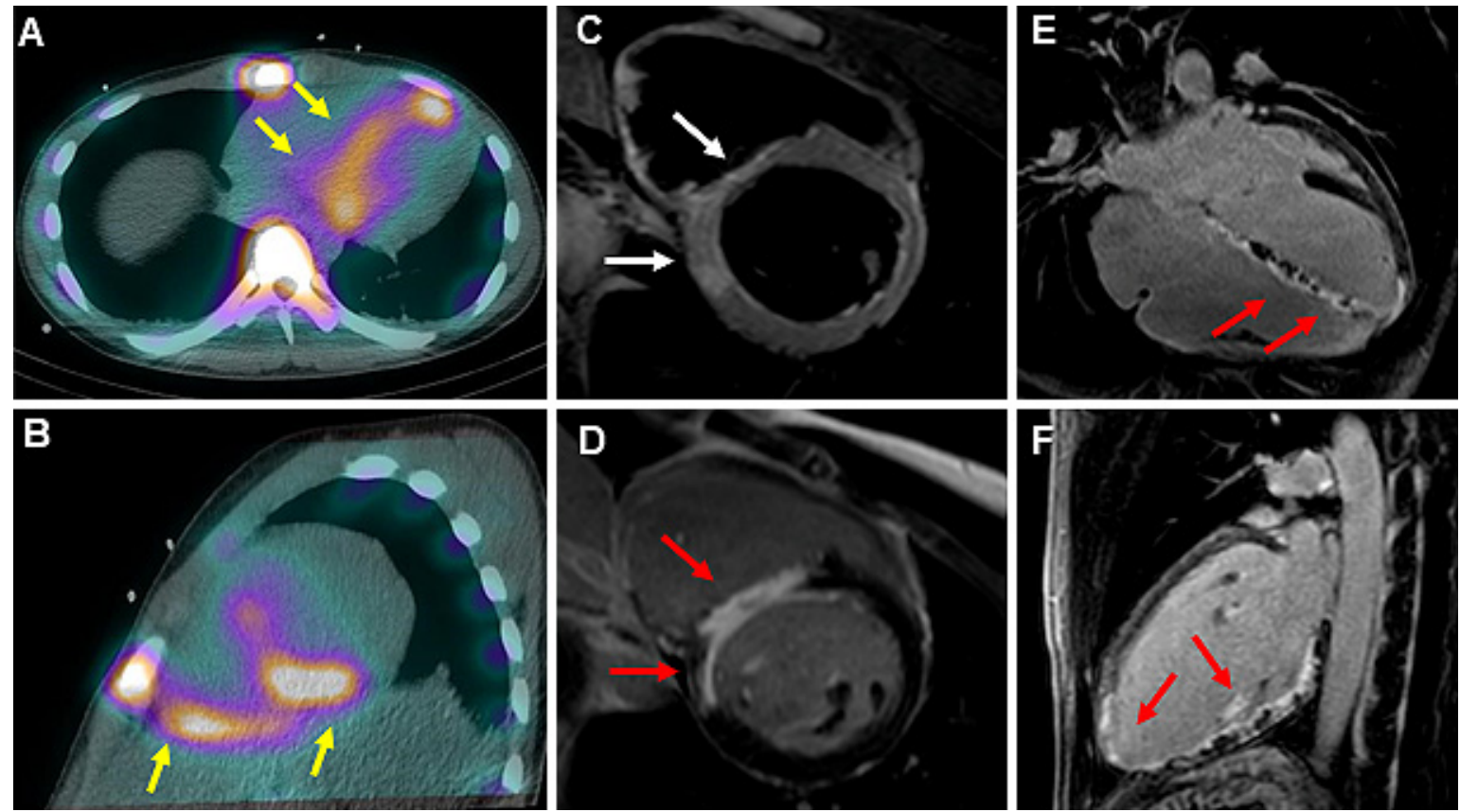

Figure 2. ${ }^{99 \mathrm{~m}}$ Tc-pyrophosphate scintigraphy (PYP) and cardiac magnetic resonance images (CMR). A and B: PYP showing significant uptake in inferior and apical wall including the right ventricle (yellow arrows). C-F: CMR, T2-weighted image showing high signal intensity in the inferoseptal wall and right ventricle (C, white arrows) and delayed enhancement in the area of myocardial infarction (D-F, red arrows).

were initiated, which successfully controlled the platelet count (platelet count 500-700 $\times 10^{9} / \mathrm{L}$ ). Under the supervision of multidisciplinary Oncocardiology Team, the patient has been asymptomatic up until the present with the continuation of a renin-angiotensin-aldosterone system inhibitor (enalapril, $10 \mathrm{mg}$ daily) and coronary vasodilators (diltiazem, $200 \mathrm{mg}$ daily; isosorbide, $40 \mathrm{mg}$ daily).

Follow-up coronary angiography with the acetylcholine provocation test was performed 12 months later after a 2-day interruption of these vasodilators. Severe multivessel vasospasm was induced by intracoronary acetylcholine infusion (Figure 3A-D). Optical coherence tomography (OCT) of the RCA suggested intimal fibrotic changes without remarkable atheromatous plaque (Figure $3 \mathrm{E})$; thus, administration of the coronary vasodilators was continued to prevent recurrence of coronary ischemia.

\section{Discussion}

We report the case of an adolescent patient with ET carrying JAK2 V617F mutation who developed acute STEMI after commencing anagrelide treatment. Persistent endothelial dysfunction was confirmed a year after through positive acetylcholine provocation test. Furthermore, intracoronary imaging demonstrated nonatheromatous intimal fibrosis possibly due to chronic endothelial damage. These underlying pathologies related to properties of ET JAK2 V617F mutation may have predisposed the patient to the fatal cardiovascular complication. Thrombotic complications in ET: The high risk of developing thrombohemorrhagic is associated with various clinical and biological factors, such as traditional cardiovascular risk factors (dyslipidemia, hypertension, smoking, diabetes, and older age), history of prior thrombosis or hemorrhage, and severe thrombocytosis. ${ }^{6,8,10)}$ Among such complications, coronary artery disease is one of the most devastating manifestations. Although relatively young patients who do not exhibit traditional coronary risk factors have also been reported to suffer from such complications, ${ }^{11-13)}$ occurrence of STEMI in patients under 20 years old is uncommon.

The mechanisms underlying acute coronary syndrome are considered to be multifactorial. Increased numbers of blood cells leading to increased blood viscosity in the context of ET promote the formation of plateletleukocyte aggregates, with concomitant release of proteases from the activated leukocytes into circulation, and consequent intravascular hypercoagulability. ${ }^{14)}$ Besides the hyperviscosity, chronic endothelial cell damage provoked by activated platelets and chronic high shear stress on the vessel wall are potentially causative factors of acute coronary syndrome. ${ }^{14)}$ Most patients with ET (approximately $60 \%$ ) harbor the JAK2 V617F mutation in their hematopoietic cells. ${ }^{2,15}$ This somatic mutation causes constitutive activation of the kinase, resulting in deregulated intracellular signaling that leads to myeloproliferative disorders, and is also known to be a significant risk factor for cardiovascular events potentially by enhancing thrombin generation in relation to platelet activity. ${ }^{2,3,713)}$ In our case, coronary endothelial cell dysfunction demonstrated by positive acetylcholine provocation test was observed a year after the onset of STEMI. We also visualized the non-atheromatous intimal fibrosis in coronary artery using OCT. These findings indicate the presence of endothelial adverse remodeling possibly attributable to chronic endothelial damage and hyperviscosity. 


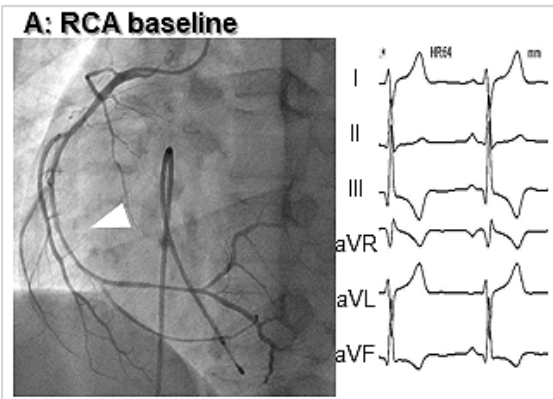

C: RCAACh $10 \mu \mathrm{g}$ IA

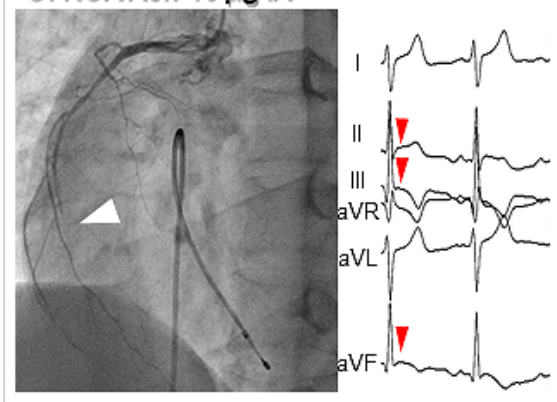

\section{B: LCA Baseline}

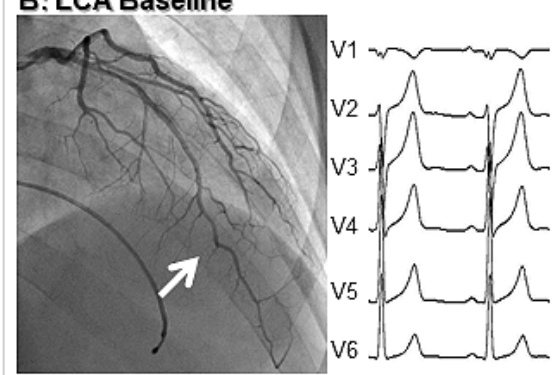

D: LCAAch $20 \mu \mathrm{g} \mathrm{IA}$

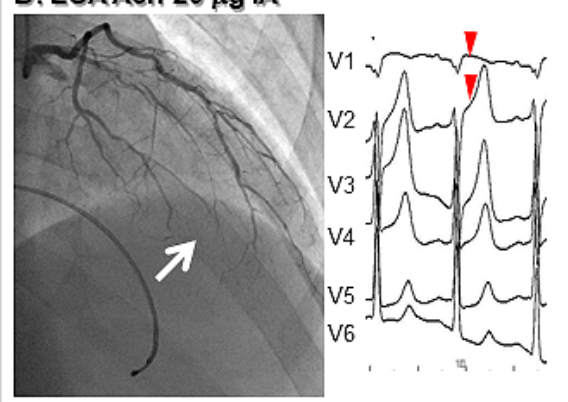

E: RCA

Cross-sectional
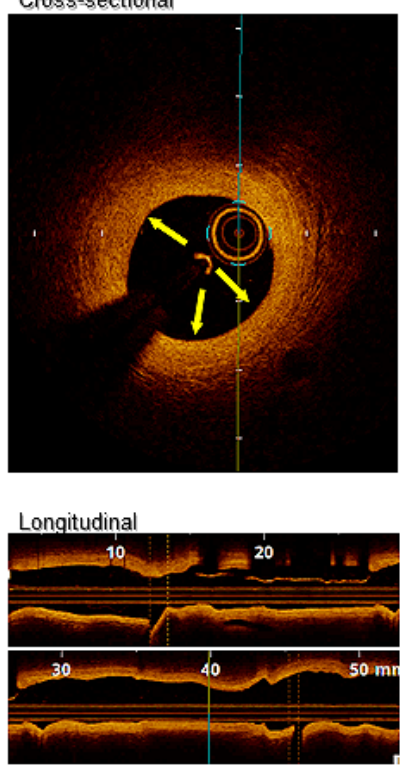

Figure 3. Follow-up coronary angiography with acetylcholine provocation test. A-D: Coronary angiogram showing non-occlusive coronary arteries at baseline (white arrowheads for the right coronary artery (RCA) and arrows for the left anterior descending artery, A and B) and total occlusion upon intracoronary acetylcholine infusion (C and D). Ischemic electrocardiographic changes (red arrowheads, fluctuating due to chest pain) corresponding to culprit arteries were simultaneously recorded. E: Optical coherence tomography of the RCA showing extensive, homogenous bright intimal thickening (yellow arrows) without prominent attenuation, suggesting fibrotic change. ${ }^{23)}$

Effect of anagrelide on cardiovascular system: Anagrelide is a selective inhibitor of thrombopoiesis. ${ }^{5,16)}$ Although the precise mechanism underlying the attenuation of platelet overproduction remains unclear, it has been postulated that the drug's effects are based on repression of transcriptional factors related to the megakaryopoiesis including GATA-1 and FOG-1 via a phosphodiesterase III (PDE III)-independent mechanism. ${ }^{17)}$ Anagrelide also inhibits cyclic adenosine monophosphate PDE III pharmacologically and has inotropic and vasodilating properties; thus, the most common cardiovascular adverse events are nonfatal manifestations, such as tachycardia/palpitations. ${ }^{4,18)}$

However, there have been some reports of acute coronary syndrome as adverse events of anagrelide in patients with ET and traditional cardiovascular risk factors. ${ }^{16,19)}$ The biological effects of anagrelide on the coronary artery are controversial; both vasospasm and vasodilation have been proposed. ${ }^{20-22)}$

In our case, multiple mechanisms including sympathetic nerve hyperactivity induced by anagrelide together with underlying endothelial condition may be involved in his coronary pathology. Although the direct association between the presence of JAK2 V617F mutation and the occurrence of AMI cannot be determined, the presence of this mutation is an important cardiovascular risk factor from the epidemiological viewpoint. Therefore, patients with ET and JAK2 V617F mutation, even without traditional cardiovascular risk factors, should be considered at high risk for coronary artery disease especially when treatment with anagrelide is necessary for cytoreduction.

\section{Conclusion}

Our adolescent patient with ET carrying JAK2 V617F mutation developed acute STEMI. Anagrelide was considered as a potential trigger, whereas persistent endothelial dysfunction and remodeling, possibly attributable to chronic endothelial damage and hyperviscosity, may be predisposing factors to his fatal cardiovascular events.

\section{Disclosure}

Authors' contributions: YG, YSu, AO, YSh, AW, KN, and TM summarized and interpreted patients' clinical data and drafted the manuscript. KF performed optimal imaging investigation and analyzed imaging data. SN and RT revised the final version of this manuscript.

Ethic approval and consent to participate and publication: For this case report, the Institutional Review Board of Saitama Medical University and International Medical Center did not require approval or consent to participate and publication.

Conflicts of interest: None.

\section{References}

1. Arber DA, Orazi A, Hasserjian R, et al. The 2016 revision to the World Health Organization classification of myeloid neoplasms and acute leukemia. Blood 2016; 127: 2391-405.

2. Tefferi A, Pardanani A. Essential thrombocythemia. N Engl J Med 2019; 381: 2135-44. 
3. Falanga A, Marchetti M. Thrombosis in myeloproliferative neoplasms. Semin Thromb Hemost 2014; 40: 348-58.

4. Gugliotta L, Tieghi A, Tortorella G, et al. Low impact of cardiovascular adverse events on anagrelide treatment discontinuation in a cohort of 232 patients with essential thrombocythemia. Leuk Res 2011; 35: 1557-63.

5. Birgegård G, Besses C, Griesshammer M, et al. Treatment of essential thrombocythemia in Europe: a prospective long-term observational study of 3649 high-risk patients in the Evaluation of anagrelide Efficacy and Long-term safety study. Haematologica 2018; 103: 51-60.

6. Tortorella G, Piccin A, Tieghi A, et al. Anagrelide treatment and cardiovascular monitoring in essential thrombocythemia. A prospective observational study. Leuk Res 2015; 39: 592-8.

7. Tefferi A, Barbui T. Personalized management of essential thrombocythemia-application of recent evidence to clinical practice. Leukemia 2013; 27: 1617-20.

8. De Stefano V, Za T, Rossi E, et al. Recurrent thrombosis in patients with polycythemia vera and essential thrombocythemia: incidence, risk factors, and effect of treatments. Haematologica 2008; 93: 372-80.

9. Fu R, Zhang L, Yang R. Paediatric essential thrombocythaemia: clinical and molecular features, diagnosis and treatment. $\mathrm{Br} \mathrm{J}$ Haematol 2013; 163: 295-302.

10. Harrison CN, Bareford D, Butt N, et al. Guideline for investigation and management of adults and children presenting with a thrombocytosis. Br J Haematol 2010; 149: 352-75.

11. Bildirici U, Celikyurt U, Ural E. Essential thrombocythemia: a case of acute ST-segment elevation myocardial infarction in a young female. Clin Cardiol 2009; 32: 104-5.

12. Lata K, Madiraju N, Levitt L. JAK2 mutations and coronary ischemia. N Engl J Med 2010; 363: 396-7.

13. Rossi C, Randi ML, Zerbinati P, Rinaldi V, Girolami A. Acute coronary disease in essential thrombocythemia and polycythemia vera. J Intern Med 1998; 244: 49-53.

14. Cella G, Marchetti M, Vianello F, et al. Nitric oxide derivatives and soluble plasma selectins in patients with myeloproliferative neoplasms. Thromb Haemost 2010; 104: 151-6.

15. Tefferi A, Guglielmelli P, Larson DR, et al. Long-term survival and blast transformation in molecularly annotated essential thrombocythemia, polycythemia vera, and myelofibrosis. Blood 2014; 124: 2507-13; quiz 615.

16. Gisslinger H, Gotic M, Holowiecki J, et al. Anagrelide compared with hydroxyurea in WHO-classified essential thrombocythemia: the ANAHYDRET Study, a randomized controlled trial. Blood 2013; 121: 1720-8.

17. Ahluwalia M, Donovan H, Singh N, Butcher L, Erusalimsky JD. Anagrelide represses GATA-1 and FOG-1 expression without interfering with thrombopoietin receptor signal transduction. J Thromb Haemost 2010; 8: 2252-61.

18. Mazzucconi MG, Redi R, Bernasconi S, et al. A long-term study of young patients with essential thrombocythemia treated with anagrelide. Haematologica 2004; 89: 1306-13.

19. Harrison CN, Campbell PJ, Buck G, et al. Hydroxyurea compared with anagrelide in high-risk essential thrombocythemia. $\mathrm{N}$ Engl J Med 2005; 353: 33-45.

20. Lin GM, Chao TY, Wang WB. Acute coronary syndromes and anagrelide. Int J Cardiol 2007; 117: e17-9.

21. Piccin A, Steurer M, Feistritzer C, et al. Observational retrospective study of vascular modulator changes during treatment in essential thrombocythemia. Transl Res J Lab Clin Med 2017; 184: 21-34.

22. Johnston H, Majewski $\mathrm{H}$, Musgrave IF. Involvement of cyclic nucleotides in prejunctional modulation of noradrenaline release in mouse atria. Br J Pharmacol 1987; 91: 773-81.

23. Prati F, Regar E, Mintz GS, et al. Expert review document on methodology, terminology, and clinical applications of optical coherence tomography: physical principles, methodology of image acquisition, and clinical application for assessment of coronary arteries and atherosclerosis. Eur Heart J 2010; 31: 401-15.

\section{Supplemental Files}

Supplemental Table

Supplemental Figures 1, 2

Please see supplemental files; https://doi.org/10.1536/ihj.20-377 